\title{
El impacto emocional del incivismo laboral y el abuso verbal en el trabajo: El papel protector de la recuperación diaria
}

\author{
Eva Garrosa ${ }^{1}$, Isabel Carmona-Cobo ${ }^{2}$, Bernardo Moreno-Jiménez ${ }^{1}$, Ana Sanz-Vergel ${ }^{3}$ \\ ${ }^{1}$ Facultad de Psicología (Universidad Autónoma de Madrid) (España) \\ ${ }^{2}$ Carrera de Psicología (Universidad Autónoma de Chile) (Chile) \\ ${ }^{3}$ Norwich Business School (University of East Anglia) (United Kingdom)
}

\begin{abstract}
Resumen: El objetivo fue estudiar el impacto diario del incivismo laboral y el abuso verbal en el bienestar emocional de los trabajadores, y explorar el papel de la recuperación. Se realizó un estudio de diario durante cinco días laborales consecutivos (525 días en total) con 105 trabajadores del sector servicios. Se evaluó en dos momentos temporales distintos, general y diario. La medida de diario consistió en la evaluación durante una semana laboral y en distintos momentos del día, mañana, tarde y noche. Los análisis multinivel mostraron resultados significativos del incivismo laboral y el abuso verbal como predictores del agotamiento emocional, y todos sobre el afecto negativo de la noche. No se encontraron relaciones entre estas variables y el afecto positivo de la noche. La recuperación durante la tarde fue clave en el estado afectivo de la noche. La relajación tuvo un efecto directo sobre el afecto negativo y las actividades enriquecedoras sobre el afecto positivo, además se encontraron efectos de moderación de la desconexión psicológica y la relajación. El diseño del estudio puede proporcionar importantes avances en medidas preventivas de la agresión en el ámbito laboral.

Palabras clave: Incivismo laboral; abuso verbal; agotamiento emocional; afecto; recuperación; estudio de diario.
\end{abstract}

\section{Introducción}

Los estudios sobre estresores laborales sitúan la agresión como uno de los factores más estresantes y frecuentes en el trabajo. Durante los últimos quince años los investigadores han generado un gran interés sobre la agresión laboral, dando lugar a una gran variedad de constructos (Hershcovis y Barling, 2010). Sin embargo, no muchos estudios analizan las consecuencias de la agresión laboral de manera diaria, ni sus repercusiones en el estado afectivo cotidiano del trabajador.

$\mathrm{El}$ incivismo laboral es una forma leve de agresión que tiene consecuencias negativas para los trabajadores y la organización. Se caracteriza por ser "una conducta antisocial de baja intensidad (ruda y descortés) con una intencionalidad ambigua de dañar a la víctima y que viola las normas laborales de respeto mutuo" (Andersson y Pearson, 1999, p. 457). A pesar de su baja intensidad, se ha comprobado que las víctimas de incivismo laboral sufren mayor estrés laboral y distracción cognitiva, y menor satisfacción laboral y creatividad, incluso pueden abandonar la organización (Lim, Cortina y Magley, 2008). Además, recientes investigaciones informan de que esta forma de agresión sutil en el trabajo representa una forma "moderna" de discriminación laboral por sexo y raza (Cortina, Kabat-Farr, Leskinen, Huerta y Magley, 2013). La Teoría del Incivismo Selectivo se basa en la idea de que el incivismo no siempre se produce de forma general, sino que

* Dirección para correspondencia [Correspondence address]:

Eva Garrosa. Calle Ivan Pavlov, 6. 28049 Madrid (España).

E-mail: eva.garrosa@uam.es
Title: Emotional impact of workplace incivility and verbal abuse at work: Daily recovery protective role.

Abstract: The aim was to study daily fluctuations from workplace incivility and verbal abuse in the emotional well-being of employees and examine the daily role of recovery. It was conducted a diary study over five consecutive working days (total of 525 days) with 105 employees. It was evaluated on two different times, general and diary moments. Diary measure was completed in a work week, three times per day, morning, afternoon and night. Multilevel modeling showed significant results for workplace incivility and verbal abuse as predictors of emotional exhaustion, as well as an impact of all these variables on negative affect at night. However, nonsignificant relationships were found for positive affect at night. Recovery from daily work was a key factor predicting affect at night. Relaxation showed main effects on negative affect at night and mastery on positive affect at night. Both psychological detachment and relaxation showed a moderating role. The present diary study of occupational health psychology helps us achieve greater knowledge in this area and contribute on the prevention of aggression in the organizational context.

Key words: Workplace incivility; verbal abuse; emotional exhaustion; affect; recovery; diary study.

constituye una expresión encubierta de discriminación generando una disfunción en muchos contextos de trabajo (Kabat-Farr y Cortina, 2012).

Otra forma de agresión muy frecuente en las organizaciones es el abuso verbal (Grandey, Dickter y Sin, 2004), especialmente en trabajos que demandan interacción, situación habitual del sector servicios. Las manifestaciones de abuso verbal pueden adoptar distintas formas a través de un rango variado de conductas agresivas (insultos, gritos, amenazas, silencios incómodos, etc.). Discernir en qué contextos se presentan estas conductas resulta relevante para alcanzar un mayor conocimiento del problema y tomar posibles medidas de prevención. Para ello, es importante prestar atención al origen de la agresión y al tipo de trabajo. Aquellas personas que trabajan en ocupaciones que requieren de una constante interacción con el otro, como sucede en el sector servicios, están expuestos a más situaciones de abuso verbal, especialmente cuando el abuso proviene de los clientes a los que se les presta el servicio (Grandey, Kern y Frone, 2007).

Los datos evidencian que el agotamiento emocional es una de las consecuencias de diferentes formas de agresión laboral (Ben-Zur y Yagil, 2005) con graves consecuencias para la salud de los trabajadores. Esta investigación estudia los efectos que pueden tener estas formas de agresión en el bienestar emocional diario de los trabajadores y se plantea la siguiente hipótesis:

Hipótesis 1. El nivel diario de incivismo laboral y abuso verbal estará positivamente relacionado con el agotamiento emocional que sienten las personas cuando salen de trabajar. 
Las respuestas generadas por la agresión laboral pueden producir estados emocionales en los trabajadores, como miedo o enfado (Schat y Kelloway, 2000). Las secuelas del estado de ánimo negativo incluyen los síntomas psicológicos y somáticos; el decremento en las actitudes positivas hacia el trabajo; un aumento en las conductas de abandono; y producen una reacción intensa en el afecto negativo. Sin embargo, esto no sucede con el afecto positivo, al ser estados de ánimo independientes (Watson, Clark y Tellegen, 1988). De igual modo, la investigación ha mostrado evidencias en la relación entre las interacciones interpersonales negativas en el trabajo y las consecuencias en el estado afectivo de los trabajadores (Dimotakis, Scott y Koopman, 2011; Xanthopoulou, Bakker, Demerouti y Schaufeli, 2009). Partiendo del impacto que parece tener la agresión laboral y el agotamiento emocional sobre el estado afectivo de los trabajadores, se propone la segunda hipótesis:

Hipótesis 2. El nivel diario de incivismo laboral, abuso verbal y agotamiento emocional al salir del trabajo estará positivamente relacionado con el afecto negativo (a) de la noche, y no tendrá relación con el afecto positivo (b) de la noche.

Se ha comprobado que la realización de actividades de desconexión psicológica, relajación, actividades enriquecedoras y control del tiempo libre ayudan a las personas a recuperarse del estrés laboral diario y mejoran su bienestar (Sonnentag, Kuttler y Fritz, 2010). Sin embargo, muchos trabajadores son incapaces de apartarse psicológicamente del trabajo cuando termina su jornada laboral. Esta incapacidad de desconexión es un mecanismo clave que contribuye a explicar la fatiga y el malestar (Sonnentag, 2011). Los procesos relacionados con la recuperación del estrés laboral son relevantes para la salud de las personas, su bienestar y su rendimiento laboral. Mas que una actividad específica que por sí misma ayude a recuperarse del estrés laboral, serían los procesos subyacentes comunes a estas actividades los que estarían interviniendo en la recuperación del trabajador (Sonnentag y Fritz, 2007). En esta línea, se plantea la siguiente hipótesis:

Hipótesis 3. El nivel diario de desconexión psicológica (a), relajación (b), actividades enriquecedoras (c) y control del tiempo libre (d) después del trabajo estará positivamente relacionado con el afecto positivo de la noche, y negativamente relacionado con el afecto negativo de la noche.

Del mismo modo, recientes investigaciones han estudiado el papel que adoptan los procesos de recuperación sobre el bienestar diario (Sanz-Vergel, Demerouti, MorenoJiménez y Mayo, 2010). Sonnentag (2011) ha planteado el Modelo de Estrés-Desconexión Psicológica que propone la recuperación como un moderador que amortigua la relación estrés-desconexión. Esto lleva a plantear la cuarta hipótesis:

Hipótesis 4. La desconexión psicológica (a), la relajación (b), las actividades enriquecedoras (c) y el control del tiempo libre (d) tendrán un papel moderador en el efecto del incivismo laboral, abuso verbal y agotamiento emocional sobre el afecto positivo y el afecto negativo de la noche.
En resumen, la investigación tiene como objetivo observar el impacto diario del incivismo laboral y el abuso verbal en el bienestar emocional diario de los trabajadores, así como explorar el papel diario de las experiencias de recuperación realizadas durante el tiempo libre de la tarde.

\section{Método}

\section{Participantes}

Se contactó con diversas entidades del sector servicios y se informó a los trabajadores la posibilidad de participar en el estudio. Los trabajadores que aceptaron participar en el estudio recibieron un sobre que incluía el protocolo de evaluación, y una carta de presentación con las instrucciones y el consentimiento informado.

Acorde con los dos momentos temporales de evaluación -general y diario-, el protocolo estaba formado por el cuestionario general y el cuestionario de diario. Los participantes respondieron el cuestionario general en un solo momento, antes de realizar el de diario. El cuestionario de diario se realizó durante una semana laboral (de lunes a viernes) y en tres momentos del día: por la mañana, antes de ir a trabajar; por la tarde, al salir del trabajo; y por la noche, antes de dormir. Los investigadores dieron instrucciones muy precisas a los participantes acerca del procedimiento de respuesta a fin de garantizar los momentos de evaluación, de suma relevancia en este estudio. Además, se contactó con los participantes vía telefónica para comprobar que se seguía el procedimiento adecuado. Igualmente, se recomendó la utilización de la alarma móvil como recordatorio de los momentos de evaluación diarios.

Se recogieron 106 protocolos (120 entregados) con consentimiento informado. De ellos, hubo que eliminar un participante que no había cumplimentado de forma correcta el protocolo. La muestra total es de 105 trabajadores del sector servicios (comercio, hostelería, tecnologías de la información, servicios a empresas y juego) de la Comunidad de Madrid, con evaluaciones diarias durante cinco días laborales consecutivos, con un total de 525 días evaluados (Nägel y Sonnentag, 2013). Teniendo en cuenta la dificultad que conlleva para los participantes este tipo de diseño, la tasa de respuesta fue elevada (87.5\%). El 60\% son mujeres y el $40 \%$ hombres, con una edad media de 35.01 años con estudios superiores. El 58.4\% tienen contrato indefinido, trabajan la mayoría en turno de mañana y tarde, desempeñando 35-40 horas semanales.

\section{Instrumentos}

Todas las variables del estudio se evaluaron de forma general y diaria, utilizándose los mismos instrumentos de evaluación para ambas medidas. Sin embargo, los participantes recibieron instrucciones diferentes para cada evaluación. Las instrucciones hacían referencia al momento concreto en el que se registraba la respuesta. Además de las variables del es- 
tudio, en el cuestionario general los participantes respondieron a variables sociodemográficas y características laborales. El género, el estrés de rol (Garrosa, Rainho, MorenoJimenez y Monteiro, 2010), el nivel general de la variable a estudiar y el afecto de la mañana fueron tomadas como va- riables control. Todos los instrumentos de evaluación mostraron adecuados índices de fiabilidad (Abad, Olea, Ponsoda y García, 2011) tanto para la medida general como para la medida de diario (Tabla 1).

Tabla 1. Fiabilidad y Correlaciones de Pearson entre las variables del estudio.

\begin{tabular}{|c|c|c|c|c|c|c|c|c|c|c|c|c|c|c|c|c|c|c|}
\hline & $M$ & $D T$ & $\alpha$ & 1 & 2 & 3 & 4 & 5 & 6 & 7 & 8 & 9 & 10 & 11 & 12 & 13 & 14 & 1516 \\
\hline Género $^{a}$ & 1.60 & .49 & - & - & & & & & & & & & & & & & & \\
\hline Estrés de Rola ${ }^{\mathrm{a}}$ & 4.30 & .99 & .87 & .05 & - & & & & & & & & & & & & & \\
\hline Agotamiento emocional ${ }^{a}$ & 2.17 & 1.39 & .90 & .01 & .07 & - & & & & & & & & & & & & \\
\hline Afecto negativo & 2.17 & .63 & .76 & $.16^{* *}$ & -.03 & $-20^{* *}$ & - & & & & & & & & & & & \\
\hline Afecto positivo & 3.83 & .53 & .80 & $.17^{* *}$ & $.19^{* *}$ & $.32^{* *}$ & $-.25^{* *}$ & - & & & & & & & & & & \\
\hline Afecto negativo mañana ${ }^{b}$ & 1.50 & .68 & .79 & .05 & .06 & $.42^{* *}$ & -07 & $.35^{* *}$ & - & & & & & & & & & \\
\hline Afecto positivo mañana ${ }^{b}$ & 3.17 & 1.03 & .89 & .03 & -.06 & $-.26^{* *}$ & $.33^{* *}$ & $-.14^{* *}$ & $-.22^{* *}$ & - & & & & & & & & \\
\hline Incivismo laboral tarde ${ }^{\mathrm{b}}$ & 1.26 & .57 & .78 & -.04 & -.03 & -.05 & $.12^{* *}$ & .06 & $.21^{* *}$ & $.07^{*}$ & - & & & & & & & \\
\hline Abuso verbal tarde ${ }^{b}$ & 1.18 & .35 & .71 & $-.09^{*}$ & -.07 & -.01 & -.02 & $.19^{* *}$ & $.13^{* *}$ & .05 & $.44^{* *}$ & - & & & & & & \\
\hline Agotamiento emocional tarde $\mathrm{e}^{\mathrm{b}}$ & 2.03 & 1.63 & .90 & $.13^{* *}$ & $.11^{*}$ & $.24^{* *}$ & $-.16^{* *}$ & $.67^{* *}$ & $.39^{* *}$ & $-.13^{* *}$ & $.23^{* *}$ & $.29^{* *}$ & - & & & & & \\
\hline Desconexión psicológica noche ${ }^{b}$ & 3.60 & 1.07 & .86 & .04 & .01 & $-.10^{*}$ & .06 & $-.28^{* *}$ & $-.10^{*}$ & -.02 & $-.16^{* *}$ & $-.23^{* *}$ & $-.46^{* *}$ & - & & & & \\
\hline Relajación noche & 3.68 & 1.09 & .86 & -.03 & .05 & $-.10^{*}$ & $.15^{* *}$ & $-.31^{* *}$ & $-.14^{* *}$ & .05 & $-.09^{*}$ & $-.18^{* *}$ & $-.45^{* *}$ & $.63^{* *}$ & - & & & \\
\hline Actividades enriquecedoras noche & 3.16 & 1.31 & .94 & $-.09^{*}$ & -.07 & -.08 & $.29^{* *}$ & $-.20^{* *}$ & .013 & $.13^{* *}$ & .05 & -.04 & $-.28^{* *}$ & $.32^{* *}$ & $.50^{* *}$ & - & & \\
\hline Control del tiempo libre noche ${ }^{b}$ & 3.91 & 1.47 & .67 & .01 & $.09^{*}$ & -.07 & $.18^{* *}$ & $-.25^{* *}$ & $-.18^{* *}$ & $.11^{*}$ & -.08 & $-.14^{* *}$ & $-.33^{* *}$ & $.39^{* *}$ & $.63^{* *}$ & $.38^{* *}$ & - & \\
\hline Afecto negativo noche $e^{b}$ & 1.44 & .70 & .81 & .05 & .03 & $.40^{* *}$ & $-.11^{*}$ & $.36^{* *}$ & $.52^{* *}$ & -.08 & $.31^{* *}$ & $.30^{* *}$ & $.45^{* *}$ & $-.25^{* *}$ & $-.31^{* *}$ & $-.17^{* *}$ & $-.26^{* *}$ & - \\
\hline Afecto positivo noche ${ }^{b}$ & 2.45 & 1.11 & .79 & $-.21^{* *}$ & $-.10^{*}$ & -.08 & $.22^{* *}$ & $-.30^{* *}$ & -.07 & $.36^{* *}$ & .03 & .05 & $-.24^{* *}$ & $.15^{* *}$ & $.13^{* *}$ & $.25^{* *}$ & $.11^{*}$ & $-.04-$ \\
\hline
\end{tabular}

$\begin{array}{lllll}\text { Afecto positivo noche } & \\ \text { a Predictores del nivel 2, person-level. }{ }^{\mathrm{b}} \text { Predictores del nivel 1, day-level. }\end{array}$

$* p<.05, * * p<.01, * * * p<.001$.

Escala de Afecto Positivo y Negativo. Los estados de ánimo positivo y negativo se evaluaron con la versión corta (Thompson, 2007) del Positive And Negative Affect Schedule (PANAS; Watson y cols., 1988). La escala mide mediante diez adjetivos, cinco para afecto positivo y cinco para afecto negativo, el estado afectivo de los participantes. Esta escala está compuesta por dos factores independientes: el afecto positivo y el afecto negativo. La bidimensionalidad del afecto medida con el PANAS (1988) es una estructura consistente, independientemente de la cultura y el tipo de país en el que se evalúe. Por ejemplo, se ha aplicado esta medida en diferentes tipos de muestras norteamericanas y en poblaciones de diferentes culturas como la japonesa (Watson y Clark, 1984), la rusa (Balatsky y Diener, 1993) y la israelí (Almagor y Ben-Porath, 1989), encontrando para todas buenos resultados. La escala de respuesta para los dos factores -positivo y negativo- es de tipo Likert, oscilando entre 1 ("Nunca me he sentido asî") y 6 ("Me siento así a diario"). Los participantes tenían que indicar en qué grado se sentían con afecto positivo (p.e. "Dispuesto", "Inspirado", "Decidido") y con afecto negativo (p.e. "Disgustado", "Hostil", "Avergonzado") según el momento de evaluación. Cuando los trabajadores respondían a la escala en el cuestionario general, se les pedía que indicaran en qué medida se sentían generalmente de ese modo; mientras que cuando respondían al cuestionario de diario, las instrucciones eran: "En este momento me siento...", para señalar el día y el momento del día en el que estaban respondiendo. Los índices de fiabilidad fueron adecuados tanto para afecto positivo $(\alpha=.80)$ como para afecto negativo $(\alpha=.76)$ en la medida general. En la medida de diario, todos los momentos de evaluación obtuvieron buenos índices, el afecto positivo $(\alpha=.89)$ de la mañana; el afecto negativo $(\alpha=.79)$ de la mañana; el afecto positivo $(\alpha$ $=.79)$ de la noche; $y$ el afecto negativo $(\alpha=.81)$ de la noche.

Escala de Incivismo Laboral (Workplace Incivility Scale) (WIS; Cortina, Magley, Williams y Langhout, 2001). Esta escala evalúa los comportamientos de incivismo que tienen lugar en el contexto de trabajo. Los participantes tenían que informar, a través de una escala tipo Likert y un rango de respuesta de 1 ("No, no es cierto") a 6 ("Si, es cierto") el grado en el que se habían encontrado en diferentes situaciones como: "me han menospreciado y/o subestimado", "han hecho comentarios humillantes o degradantes sobre mi", "he sido ignorado o excluido del resto de compañeros o grupo profesional", por citar algunos ejemplos. El cuestionario general guiaba al participante para responder según las siguientes instrucciones: "Durante el tiempo que llevas trabajando en tu actual empresa, te has encontrado en situaciones en las que alguno de tus superiores o compañeros...". Por el contrario, las instrucciones de la escala evaluada por la tarde después de salir del trabajo, en el cuestionario de diario, comenzaba: "Hoy me he encontrado en situaciones en las que alguno de mis superiores o compañeros...". En ambos cuestionarios, general y diario, se encontraron buenos índices de fiabilidad. Para la medida general se obtuvo un coeficiente alpha de Cronbach de .90; y para la medida de diario, un .78. Teniendo en cuenta los criterios de los expertos que recomiendan un punto crítico a partir de .70 (Abad y cols., 2011), a pesar de que el obtenido en el cuestionario de diario sea menor que el general, ambos indicadores muestran buenos coeficientes.

Escala de Conflicto Interpersonal en el Trabajo (Interpersonal Conflict at Work Scale) (ICWS; Spector y Jex, 1998). La escala registra las conductas de abuso verbal en el trabajo. Esta medida indica el grado en el que jefes, compañeros y 
clientes muestran comportamientos de abuso usando una escala tipo Likert (de 1 "Nunca o menos de una vez al día" a 6 "Varias veces al día"). Se utilizaron tres ítems que evalúan la frecuencia con la que habían gritado, habían discutido y habían sido groseros con el trabajador. La escala medida en el cuestionario general se presentaba de la siguiente forma: "Indica la frecuencia con la que se dan las siguientes situaciones en tu trabajo, teniendo en cuenta la escala señalada... ¿Con qué frecuencia te han gritado?’. La del cuestionario de diario, a diferencia, se presentaba como sigue: "¿Con qué frecuencia te han gritado hoy en el trabajo?”. En ambos casos, los participantes respondían la frecuencia de estos comportamientos por parte de jefes, compañeros y clientes. El coeficiente de fiabilidad para la escala general fue de .77, y para la escala de diario fue .71 .

Escala de Agotamiento Emocional asociado al Trabajo (Scale of Job-Related Exhaustion) (SJRE; Wharton, 1993). La escala medida en el cuestionario general está compuesta por seis ítems que miden de 1 ("Nunca me he sentido así") a 6 ("Me siento así a diario") la frecuencia con la que los participantes experimentan generalmente diversas emociones derivadas del trabajo (p.e. "Me siento frustrado por mi trabajo", "Me siento exhausto al final de mi jornada laboral"). Las instrucciones eran: "Señala con una $\mathrm{X}$ la frecuencia como te sientes actualmente en cada una de las siguientes situaciones...". En esta medida, la escala mostró un alto índice de fiabilidad, siendo el alpha de Cronbach de .90. Respecto a la escala incluida en el cuestionario de diario, por la tarde al salir del trabajo, los participantes informaban cómo se sentían "Hoy" para cada uno de los seis ítems. La escala de respuesta oscila entre 1 ("No, no es cierto") y 6 ("Si, es cierto"). El coeficiente de fiabilidad que se obtuvo en esta medida fue de .89. Se optó por el uso de esta escala debido a su especificidad en la evaluación de comportamientos derivados del trabajo, a diferencia de otras escalas que hacen referencia a la dimensión de agotamiento emocional como parte del síndrome de burnout o desgaste profesional (Maslach y Jakson, 1986).

Cuestionario de Experiencias de Recuperación (SanzVergel y cols., 2010). Se usó la adaptación española del Recovery Experience Questionnaire (REQ; Sonnentag y Fritz, 2007) para evaluar la recuperación del estrés laboral. El instrumento evalúa los procesos psicológicos subyacentes que ayudan al trabajador a recuperarse de situaciones estresantes vinculadas con el trabajo. La escala total se compone de dieciséis ítems y se subdivide en cuatro experiencias de recuperación: desconexión psicológica (p.e. "Cuando he salido de trabajar, me he olvidado completamente de mi trabajo”), relajación (p.e. "He realizado actividades que me ayudan a sentirme relajado"), actividades enriquecedoras (p.e. "Fuera del trabajo busco nuevos retos intelectuales") y control del tiempo libre (p.e. "He decidido por mí mismo cómo pasar mi tiempo libre"). Siguiendo las instrucciones del cuestionario evaluado en la medida general, los participantes tenían que indicar con una cruz la alternativa que mejor se ajustara al modo de ser o actuar, con una escala de respuesta tipo Likert, de 1 "Total- mente en desacuerdo" a 6 "Totalmente de acuerdo". En este caso, el coeficiente de fiabilidad para todos los ítems fue .91. En cada una de las dimensiones se obtuvieron los siguientes índices: .76 para la desconexión psicológica; 80 para la relajación; .93 para las actividades enriquecedoras; y .93 para el control del tiempo libre. En relación al uso del cuestionario para la medida de diario, se evaluó la recuperación por la noche, antes de irse a dormir. En este momento se preguntaba a los trabajadores en qué grado "Hoy" habían sido capaces de recuperarse, a través de una escala de respuesta entre 1 "No, no es cierto" y 6 "Si, es cierto". Todos los coeficientes mostraron adecuados índices de fiabilidad, tanto para el cuestionario con los dieciséis ítems $(\alpha=.89)$, como para la desconexión psicológica $(\alpha=.85)$, la relajación $(\alpha=.86)$, las actividades enriquecedoras $(\alpha=.93)$, y el control del tiempo libre $(\alpha=.67)$. Este último obtuvo el coeficiente más bajo; sin embargo, teniendo en cuenta que solo se compone de cuatro items y que el índice de la escala total es alto, se considera un coeficiente adecuado (Abad y cols., 2011).

\section{Metodología y análisis de datos}

Se trata de un estudio de diario, de dos niveles: el nivel 1 (i), día (day-level); y el nivel 2 (j), sujeto (person-level). El nivel 1 incluye las variables evaluadas de forma diaria mediante el cuestionario de diario. Esto permitió observar el cambio individual en cada participante para cada una de las variables, según el día (de lunes a viernes) y el momento de evaluación (mañana, tarde, noche). El nivel 2 recoge las variables evaluadas de forma general, a través del cuestionario de evaluación general. Este nivel de medida permitió establecer los niveles generales (trait) de cada participante en las variables evaluadas. Por otro lado, el afecto fue utilizado como variable control atendiendo al nivel de medida. De este modo, los análisis multinivel incluyen el control del nivel general de afecto (trait) en el modelo 1 para la medida general, y el control del nivel diario de la mañana en el modelo 2 , como control diario.

Se obtuvo el coeficiente de correlación intraclase para cada variable criterio. El $25.19 \%$ de la varianza fue explicado por las fluctuaciones a nivel diario para el agotamiento emocional de la tarde; el $45.60 \%$ de la varianza para el afecto negativo de la noche, y el $43.68 \%$ de la varianza para el afecto positivo de la noche. Incluso, todas las variables predictoras obtuvieron índices que justifican el uso del análisis multinivel (Hox y Robers, 2011). El porcentaje de variación intra-sujeto para cada variable atendiendo al momento de evaluación fue: $47 \%$ afecto positivo y $44 \%$ afecto negativo de la mañana; $47 \%$ incivismo laboral, $36 \%$ abuso verbal, y $25 \%$ agotamiento emocional de la tarde; $49 \%$ desconexión psicológica, $42 \%$ relajación, 29\% actividades enriquecedoras, y 53\% control del tiempo libre de la noche.

Teniendo en cuenta este diseño y la estructura de los datos se aplicó la aproximación de modelos jerárquicos lineales (Bryk y Raudenbush, 1992) utilizando el software MlwiN (Rashbash, Browne, Healy, Cameron y Charlton, 2000). Con 
el fin de evitar la multiconealidad, las variables del nivel 2 se centraron en torno a las puntuaciones medias de todos los sujetos y las del nivel 1 aproximando las puntuaciones a la media de cada sujeto individualmente (Ohly, Sonnentag, Niessen y Zapf, 2010).

\section{Resultados}

Los análisis multinivel para cada variable criterio se realizaron de forma específica y simultánea para el nivel general (nivel 1, day-level) y el nivel diario (nivel 2, person-level) con los respectivos momentos de evaluación. Las variables se introdujeron por modelos. Para las tres variables criterio, agotamiento emocional, afecto negativo y afecto positivo, la constante se muestra en el null model; el modelo 1 añade las variables control del nivel general (género, estrés de rol y nivel general de la variable criterio); el modelo 2 introduce las variables control del nivel diario (afecto positivo y negativo de la mañana); el modelo 3 añade las formas de agresión laboral estudiadas (incivismo laboral y abuso verbal de la tarde). Para el afecto negativo y el afecto positivo se añadieron ade- más, el modelo 4 que introduce el agotamiento emocional; el modelo 5 con las cuatro experiencias de recuperación (desconexión psicológica, relajación, actividades enriquecedoras, control del tiempo libre); y un último modelo con las interacciones entre la recuperación, las formas de agresión laboral, y el agotamiento emocional.

El análisis multinivel del agotamiento emocional de la tarde (Tabla 2) muestra en el primer modelo un ajuste significativo sobre el null model, donde se incluye la constante (difference of $-2 \times \log =90.776, g l=3, p<.001$ ), debido a la significación del nivel general de agotamiento emocional $(t=$ $11.558, p<.001)$. El modelo 2 muestra un buen ajuste (difference of $-2 \times \log =9.106, g l=2, p<.01)$ por el resultado significativo del afecto negativo de la mañana $(t=2.833, p<.05)$. El modelo 3 incluye la agresión laboral y muestra el mejor nivel de ajuste (difference of $-2 \times \log =42.634, g l=2, p<.001$ ) con la significación del incivismo laboral $(t=2.219, p<.05)$ y el abuso verbal $(t=3.665, p<.01)$. En base a estos resultados, podemos mantener la hipótesis 1 , ya que son las variables de agresión laboral las predictoras del agotamiento emocional de la tarde.

Tabla 2. Resultados multinivel de las variables predictoras del agotamiento emocional de la tarde.

\begin{tabular}{|c|c|c|c|c|c|c|c|c|c|c|c|c|}
\hline \multirow{2}{*}{ Variables } & \multicolumn{3}{|c|}{ Null Model } & \multicolumn{3}{|c|}{ Modelo 1} & \multicolumn{3}{|c|}{ Modelo 2} & \multicolumn{3}{|c|}{ Modelo 3} \\
\hline & Coeficiente & SE & $t$ & Coeficiente & SE & $t$ & Coeficiente & SE & $t$ & Coeficiente & SE & $t$ \\
\hline Constante & 2.035 & .1421 & 4.330 & 2.035 & .092 & 22.119 & 2.035 & .092 & 22.119 & 2.044 & .092 & 22.217 \\
\hline Género & & & & -.007 & .010 & -.7 & -.007 & .010 & -.7 & -.007 & .010 & -.7 \\
\hline Estrés de Rola & & & & -.003 & .096 & -.031 & -.003 & .096 & -.031 & -.006 & .096 & -.062 \\
\hline Agotamiento emocionala & & & & .786 & .068 & $11.558^{* * *}$ & .786 & .068 & $11.558^{* *}$ & .782 & .068 & $11.5^{* *}$ \\
\hline Afecto positivo mañana ${ }^{b}$ & & & & & & & -.022 & .057 & -.385 & -.049 & .057 & -.859 \\
\hline Afecto negativo mañanab & & & & & & & .255 & .090 & $2.833^{*}$ & .171 & .090 & 1.9 \\
\hline Incivismo laboral tarde ${ }^{\mathrm{b}}$ & & & & & & & & & & .233 & .105 & $2.219 *$ \\
\hline Abuso verbal tardeb & & & & & & & & & & .733 & .200 & $3.665^{* *}$ \\
\hline$-2 X \log (l h)$ & \multirow{3}{*}{\multicolumn{3}{|c|}{1562.911}} & \multicolumn{3}{|c|}{1472.135} & \multicolumn{3}{|c|}{1463.029} & \multicolumn{3}{|c|}{1420.395} \\
\hline Difference of $-2 X \log$ & & & & \multicolumn{3}{|c|}{$90.776^{* * *}$} & \multicolumn{3}{|c|}{$9.106^{* *}$} & \multicolumn{3}{|c|}{$42.634 * * *$} \\
\hline$D f$ & & & & \multicolumn{3}{|c|}{3} & \multicolumn{3}{|c|}{2} & \multicolumn{3}{|c|}{2} \\
\hline Level 1 intercept variance (SE) & \multicolumn{3}{|c|}{$.668(.046)$} & \multicolumn{3}{|c|}{$.668(.046)$} & \multicolumn{3}{|c|}{$.654(.025)$} & \multicolumn{3}{|c|}{$.623(.043)$} \\
\hline Level 2 intercept variance (SE) & \multicolumn{3}{|c|}{$1.983(.292)$} & \multicolumn{3}{|c|}{$.758(.124)$} & \multicolumn{3}{|c|}{$.761(.123)$} & \multicolumn{3}{|c|}{$.759(.123)$} \\
\hline
\end{tabular}

aPredictores del nivel 2, person-level. bPredictores del nivel 1, day-level. $* p<.05,{ }^{* *} p<.01, * * * p<.001$.

En los resultados multinivel del afecto negativo de la noche (Tabla 3), el primer modelo muestra un ajuste significativo (difference of $-2 \times \log =30.503, g l=3, p<.001$ ). El modelo 2 muestra un ajuste significativo (difference of $-2 \times \log =$ $5.362, g l=2, p<.05)$ dada la significación del afecto negativo de la mañana $(t=2.28, p<.05)$. El modelo 3 muestra el mejor nivel de ajuste (difference of $-2 \times \log =40.252, \mathrm{gl}=2, p$ $<$.001) y las dos variables introducidas han resultado significativas, el incivismo laboral $(t=2.73, p<.05)$ y el abuso verbal $(t=3.86, p<.05)$ como predictoras del afecto negativo de la noche. El modelo 4 mantiene la significación del modelo previo y añade el resultado significativo del agota- miento emocional $(t=4.14, p<.05)$ con un buen ajuste (difference of $-2 \times \log =17.549, g l=1, p<.05)$. Según este modelo, el incivismo laboral, abuso verbal y agotamiento emocional serían predictores del afecto negativo de la noche. El último modelo introduce la recuperación y muestra un elevado nivel de ajuste (difference of $-2 \times \log =23.95, g l=4, p<.001$ ). La agresión se mantiene significativa y a ello se añade la relajación $(t=-2.82, p<.05)$ como experiencia de recuperación. Por lo tanto, según los resultados multinivel, podemos mantener las hipótesis $2 \mathrm{a}$, y $3 \mathrm{~b}$ para el afecto negativo de la noche. 
Tabla 3. Resultados multinivel de las variables predictoras del afecto negativo de la noche.

\begin{tabular}{|c|c|c|c|c|c|c|c|c|c|c|c|c|c|c|c|c|}
\hline \multirow[b]{2}{*}{ Variables } & Null Model & \multicolumn{3}{|c|}{ Modelo 1} & \multicolumn{3}{|c|}{ Modelo 2} & \multicolumn{3}{|c|}{ Modelo 3} & \multicolumn{3}{|c|}{ Modelo 4} & \multicolumn{3}{|c|}{ Modelo 5} \\
\hline & $\begin{array}{l}\text { Coefi- } \\
\text { ciente }\end{array}$ & $\begin{array}{l}\text { Coefi- } \\
\text { ciente }\end{array}$ & SE & $t$ & $\begin{array}{l}\text { Coefi- } \\
\text { ciente }\end{array}$ & $S E$ & $t$ & $\begin{array}{l}\text { Coefi- } \\
\text { ciente }\end{array}$ & $S E$ & $t$ & $\begin{array}{l}\text { Coefi- } \\
\text { ciente }\end{array}$ & SE & $t$ & $\begin{array}{l}\text { Coefi- } \\
\text { ciente }\end{array}$ & SE & $t$ \\
\hline Constante & 1.439 .05426 .64 & 1.439 & .047 & 30.61 & 1.439 & .047 & 30.61 & 1.441 & .047 & 30.02 & 1.441 & .047 & 30.65 & 1.441 & .047 & 30.65 \\
\hline Géneroa $^{a}$ & & .001 & .005 & .2 & .001 & .005 & .2 & .001 & .005 & .2 & .001 & .005 & .2 & .001 & .005 & .2 \\
\hline Estrés de Rola & & .006 & .047 & .12 & .006 & .047 & .12 & .005 & .047 & .10 & .006 & .047 & .12 & .006 & .047 & .12 \\
\hline Afecto negativo & & .439 & .074 & $5.93^{* *}$ & .439 & .074 & $5.93^{*}$ & .438 & .075 & $5.84^{*}$ & .437 & .075 & $5.82^{*}$ & .437 & .0755 & $5.82^{* *}$ \\
\hline Afecto positivo mañana ${ }^{b}$ & & & & & .033 & .033 & 1 & .018 & .033 & .54 & .024 & .032 & .75 & .028 & .032 & .87 \\
\hline Afecto negativo mañana ${ }^{\mathrm{b}}$ & & & & & .119 & .052 & $2.28^{*}$ & .061 & .052 & .81 & .041 & .051 & .80 & .056 & .050 & 1.12 \\
\hline Incivismo laboral tarde ${ }^{\mathrm{b}}$ & & & & & & & & .164 & .060 & $2.73^{*}$ & .137 & .059 & $2.32^{*}$ & .142 & .058 & $2.44 *$ \\
\hline Abuso verbal tardeb & & & & & & & & .445 & .115 & $3.86^{*}$ & .360 & .115 & $3.13 *$ & .311 & .112 & $2.77 *$ \\
\hline Agotamiento emocional tarde ${ }^{\mathrm{b}}$ & & & & & & & & & & & .116 & .028 & $4.14^{*}$ & .056 & .030 & 1.86 \\
\hline Desconexión psicológica noche & & & & & & & & & & & & & & -.049 & .035 & -1.4 \\
\hline Relajación noche ${ }^{b}$ & & & & & & & & & & & & & & -.089 & .039 & $-2.82^{*}$ \\
\hline Actividades enriquecedoras noche & & & & & & & & & & & & & & -.055 & .033 & -1.66 \\
\hline Control del tiempo libre noche ${ }^{b}$ & & & & & & & & & & & & & & -.016 & .022 & -.72 \\
\hline$-2 X \log (l b)$ & 904.814 & & 874.31 & & & 868.94 & & & $328.69^{7}$ & & & 311.148 & & & 87.198 & \\
\hline Difference of $-2 X \log$ & & & $0.503^{*}$ & & & $5.362^{*}$ & & & $0.252^{* *}$ & & & $17.549 *$ & & & $3.95^{* * *}$ & \\
\hline$D f$ & & & 3 & & & 2 & & & 2 & & & 1 & & & 4 & \\
\hline Level 1 intercept variance (SE) & $.223(.015)$ & & $23(.01$ & & & $20(.01$ & & & $07(.01$ & & & $99(.01$ & & & $37(.01$ & \\
\hline Level 2 intercept variance (SE) & $.266(.043)$ & & $87(.03$ & & & $88(.03$ & & & $89(.03$ & & & $92(.03$ & & & $3(.03$ & \\
\hline
\end{tabular}

aPredictores del nivel 2, person-level. ${ }^{b}$ Predictores del nivel 1, day-level.

$* p<.05,{ }^{* *} p<.01, * * * p<.001$.

Como se aprecia en la Tabla 4 del afecto positivo de la noche, el modelo 1 muestra un ajuste significativo (difference of $-2 \times \log =9.194, g l=3, p<.01)$. El modelo 2 ha mostrado un ajuste significativo (difference of $-2 \times \log =5.62, \mathrm{gl}=2, p<$ $.05)$ aunque menor al del modelo 1 y el afecto positivo de la mañana parece ser una variable predictora significativa $(t=$ $2.25, p<.05)$. El modelo 3 incluye la agresión laboral, muestra un ajuste significativo superior al modelo 2 (difference of -2 $x \log =21.733, g l=2, p<.01)$ debido al nivel general de afecto positivo $(t=2.62, \mathrm{p}<.05)$ y al afecto positivo de la mañana $(t=2.25, p<.05)$, sin embargo la agresión laboral no ha sido significativa. El modelo 4 , que añade el agotamiento emocional, no resultó significativo (difference of $-2 \times \log$ $=1.645, g l=1, n s)$. Finalmente, el modelo 5 muestra un ajuste significativo (difference of $-2 \times \log =17.047, \mathrm{gl}=4, p<$ $.001)$ donde las actividades enriquecedoras $(t=3.09, p<.05)$ resultaron significativas, mientras que el afecto positivo de la mañana no mostró significación. Según los resultados, se mantienen las hipótesis $2 \mathrm{~b}$, y $3 \mathrm{c}$ para el afecto positivo de la noche.

Tabla 4. Resultados multinivel de las variables predictoras del afecto positivo de la noche.

\begin{tabular}{|c|c|c|c|c|c|c|c|c|c|c|c|c|c|c|c|c|c|c|}
\hline \multirow[b]{2}{*}{ Variables } & \multicolumn{3}{|c|}{ Null Model } & \multicolumn{3}{|c|}{ Modelo 1} & \multicolumn{3}{|c|}{ Modelo 2} & \multicolumn{3}{|c|}{ Modelo 3} & \multicolumn{3}{|c|}{ Modelo 4} & \multicolumn{3}{|c|}{ Modelo 5} \\
\hline & $\begin{array}{l}\text { Coefi- } \\
\text { ciente }\end{array}$ & SE & $t$ & $\begin{array}{l}\text { Coefi- } \\
\text { ciente }\end{array}$ & SE & $t$ & $\begin{array}{l}\text { Coefi- } \\
\text { ciente }\end{array}$ & SE & $t$ & $\begin{array}{l}\text { Coefi- } \\
\text { ciente }\end{array}$ & $S E$ & $t$ & $\begin{array}{l}\text { Coefi- } \\
\text { ciente }\end{array}$ & SE & $t$ & $\begin{array}{l}\text { Coefi- } \\
\text { ciente }\end{array}$ & SE & $t$ \\
\hline Constante & 2.453 & .088 & 27.87 & 2.453 & .084 & 29.20 & 2.453 & .084 & 29.20 & 2.451 & .085 & 28.83 & 2.450 & .085 & 28.82 & 2.451 & .085 & 28.83 \\
\hline Género $^{\mathrm{a}}$ & & & & -.000 & .009 & 0 & -.000 & -.100 & .08 & -.000 & .009 & 0 & -.000 & .009 & 0 & -.000 & .009 & 0 \\
\hline Estrés de Rola ${ }^{\mathrm{a}}$ & & & & -.100 & .084 & -1.19 & -.100 & .084 & -1.19 & -.103 & .085 & -1.21 & -.104 & .085 & -1.22 & -.104 & .085 & -1.22 \\
\hline Afecto positivo $^{a}$ & & & & .447 & .159 & $2.81 *$ & .447 & .159 & $2.81 *$ & .425 & .162 & $2.62^{*}$ & .425 & .162 & $2.62 *$ & .425 & .162 & $2.62^{*}$ \\
\hline Afecto positivo mañana ${ }^{b}$ & & & & & & & .117 & .052 & $2.25^{*}$ & .117 & .052 & $2.25^{*}$ & .117 & .052 & $2.25^{*}$ & .098 & .052 & 1.88 \\
\hline Afecto negativo mañana ${ }^{b}$ & & & & & & & .106 & .081 & 1.30 & .090 & .083 & 1.08 & .088 & .084 & 1.04 & .058 & .083 & .69 \\
\hline Incivismo laboral tarde & & & & & & & & & & .014 & .097 & .14 & .013 & .098 & .13 & -.014 & .096 & -.14 \\
\hline Abuso verbal tarde ${ }^{b}$ & & & & & & & & & & -.158 & .185 & -.854 & -.164 & .188 & -.87 & -.119 & .186 & -.63 \\
\hline $\begin{array}{l}\text { Agotamiento emocional } \\
\text { tarde }\end{array}$ & & & & & & & & & & & & & .008 & .046 & .17 & .084 & .049 & 1.71 \\
\hline $\begin{array}{l}\text { Desconexión psicológica } \\
\text { noche }\end{array}$ & & & & & & & & & & & & & & & & .073 & .057 & 1.28 \\
\hline Relajación nocheb & & & & & & & & & & & & & & & & .060 & .065 & .92 \\
\hline $\begin{array}{l}\text { Actividades enriquecedoras } \\
\text { noche }\end{array}$ & & & & & & & & & & & & & & & & .170 & .055 & $3.09 *$ \\
\hline $\begin{array}{l}\text { Control del tiempo libre } \\
\text { noche }^{\text {b }}\end{array}$ & & & & & & & & & & & & & & & & -.042 & .036 & -1.16 \\
\hline$-2 X \log (l b)$ & & 1379.868 & & & 70.674 & & & 1365.054 & & & 343.23 & & & 341.58 & & & 324.539 & \\
\hline Difference of $-2 X \log$ & & & & & $194 * *$ & & & $5.62 *$ & & & $1.733^{*}$ & & & 1.645 & & & $.047^{* *}$ & \\
\hline$D f$ & & & & & 3 & & & 2 & & & 2 & & & 1 & & & 4 & \\
\hline Level 1 intercept variance (SE) & & $43(.037$ & & & $3(.037)$ & & & $536(.037)$ & & & $34(.03$ & & & $35(.03$ & & & $3(.036$ & \\
\hline Level 2 intercept variance (SE) & & $00(.112$ & & & $2(.102)$ & & & $634(.102)$ & & & $47(.10$ & & & $49(.10$ & & & $55(.105$ & \\
\hline
\end{tabular}

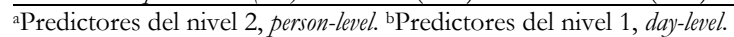

$* p<.05, * * p<.01, * * * p<.001$. 


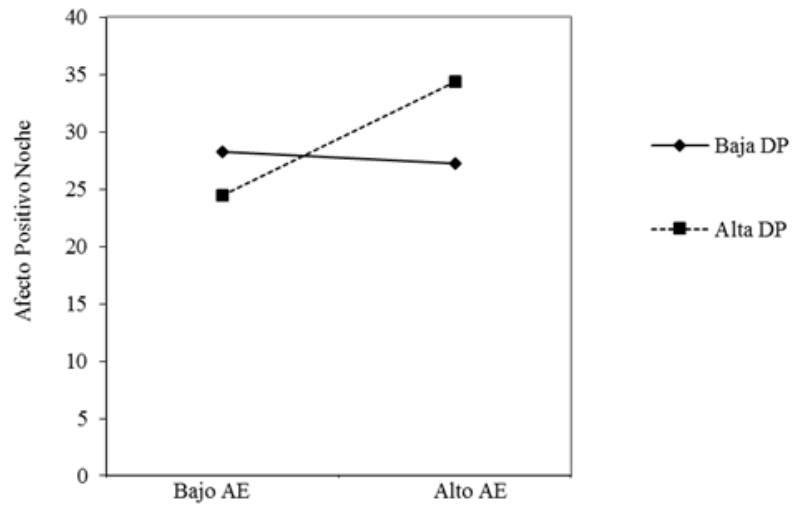

Figura 1. Interacción entre la desconexión psicológica y el agotamiento emocional sobre el afecto positivo de la noche.

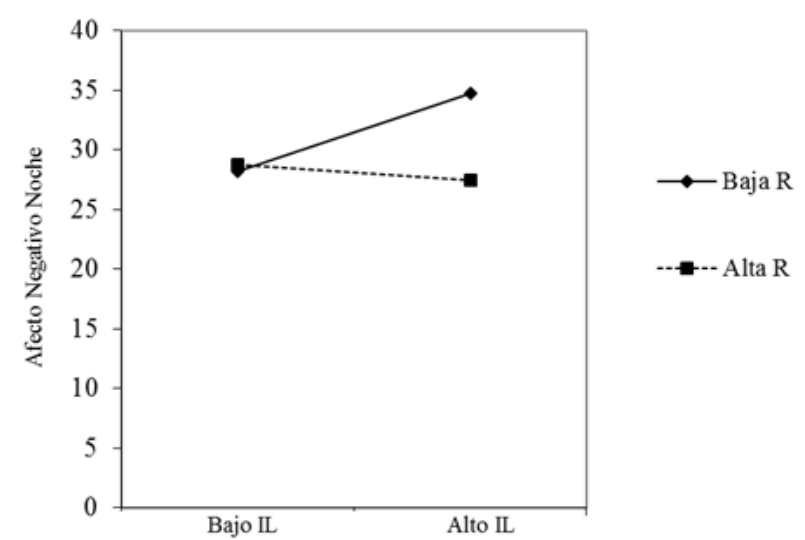

Figura 2. Interacción entre la relajación y el incivismo laboral sobre el afecto negativo de la noche.

Se añadió un sexto modelo en los análisis multinivel del afecto de la noche para contrastar la cuarta hipótesis. En relación a las interacciones del afecto positivo de la noche, se encontró significación entre la desconexión psicológica y el agotamiento emocional $(t=2.74, p<.05)$ (Figura 1). Parece que desconectar mentalmente del día de trabajo juega un papel moderador disminuyendo el efecto negativo del agotamiento emocional sobre el afecto positivo de la noche. Respecto al afecto negativo de la noche, se encontró una interacción significativa entre la relajación y el incivismo laboral $(t=-1.98, p<.05)$ (Figura 2). Esto parece indicar que los trabajadores que consiguen relajarse durante la tarde después del trabajo, se protegen del impacto negativo emocional provocado por el incivismo laboral experimentado durante la jornada laboral.

\section{Discusión}

El objetivo del presente estudio era observar el impacto diario de algunas formas de agresión laboral y el papel diario de las experiencias de recuperación en el bienestar emocional de los trabajadores del sector servicios. Los resultados del estudio muestran la importancia que tiene el entorno laboral y las consecuencias negativas que se pueden derivar de una organización tóxica para el bienestar del trabajador, de manera diaria. Según estos resultados, y de acuerdo con otros autores (Andersson y Pearson, 1999), las personas que en su lugar de trabajo experimentan conductas incívicas parecen tener mayores niveles diarios de agotamiento al salir del trabajo. Así mismo, un trabajador que está bajo situaciones de abuso verbal se sentirá con menos energía y más agotamiento físico y mental cuando sale del trabajo. Este tipo de situaciones laborales pueden ser perjudiciales no solamente para la salud del trabajador, sino para la propia organización (Dimotakis y cols., 2011). De este modo, las condiciones laborales caracterizadas por comportamientos agresivos pueden convertirse en un factor de riesgo para la persona y la organización, actuando además como una fuente potencial de discriminación hacia las mujeres (Cortina y cols, 2013). No obstante, son escasas las investigaciones que estudian el efecto diario de estas formas de agresión en el bienestar del trabajador.

¿Cómo influyen estas formas de agresión en el estado afectivo de los trabajadores al final del día? La respuesta a esta pregunta nos puede dar las claves para conocer hasta qué punto nuestra vida laboral está repercutiendo en nuestro bienestar personal. Esta investigación pretende observar el impacto emocional que tiene para un trabajador sufrir situaciones diarias de agresión laboral como las contempladas en la presente investigación. En este estudio se observa que sufrir una situación de agresión laboral a lo largo del día de trabajo influye en el estado de ánimo diario de la noche, haciendo que la persona se sienta por la noche con un estado de ánimo negativo caracterizado por enfado, disgusto, miedo y nerviosismo (Watson y cols., 1988). Sin embargo, esto no sucede con el afecto positivo de la noche. En la misma línea que Dimotakis y cols., (2011), los resultados confirman la independencia entre ambos estados afectivos y muestran la importancia de variables positivas como el afecto positivo de la mañana sobre el afecto positivo de la noche.

Recuperarse del estrés laboral diario puede ser un factor protector para el trabajador que tiene un efecto directo y moderador en el bienestar. No obstante, no todas las experiencias tienen el mismo efecto. Relajarse durante la tarde después de salir del trabajo de ocio reduce el afecto negativo en la noche y disminuye el impacto emocional negativo del incivismo laboral sufrido en el trabajo. Una vez más se observan los beneficios de la relajación sobre el bienestar personal (Hahn, Binnewies, Sonnentag y Mojza, 2011). Además, realizar actividades enriquecedoras durante la tarde libre potencia el afecto positivo de la noche al crear nuevas competencias y recursos personales, haciendo que la persona se llene de energía (Sonnentag y Fritz, 2007). Potenciar este contexto de desarrollo es un ingrediente fundamental en el marco de la psicología positiva (Ryff y Singer, 2008). En línea con Sonnentag (2011), la desconexión psicológica modera el efecto negativo del agotamiento emocional. Las personas que desconectan mentalmente del trabajo por la tarde ven 
incrementado su afecto positivo durante la noche. Igualmente, la relajación modera la relación entre incivismo laboral y afecto negativo de la noche, sobre el que tiene un efecto positivo reduciendo el impacto emocional.

La investigación, un estudio de diario con la realización de análisis multinivel, ha permitido observar las variaciones estudiando los pensamientos, sentimientos y comportamientos que una persona experimenta de forma diaria y a lo largo de un mismo día (observaciones intraindividuales), teniendo en cuenta el contexto natural de trabajo (Ohly y cols., 2010). Sin embargo, el estudio tiene limitaciones. Es una muestra del sector servicios que abarca un amplio rango de profesiones y sería conveniente controlar el tipo de área estudiada para comparar entre distintos tipos de ocupaciones. Los instrumentos de evaluación son medidas de autoinforme, sería relevante obtener medidas objetivas como registros psicofisiológicos para observar si existe correspondencia entre ambos tipos de datos, especialmente porque las variables evaluadas son de carácter emocional. Además, realizar una segunda medición aportaría mayor control y calidad de los resultados.

El presente estudio ofrece diversas aportaciones al conocimiento de la literatura sobre salud laboral controlando las variaciones diarias y las consecuencias diarias sobre el bienestar emocional. Estudia formas de agresión como el incivismo laboral y el abuso verbal que tienen importantes consecuencias a largo plazo para la organización y los trabajadores (Lim y cols., 2008). Esta investigación pone de manifiesto el impacto emocional diario de estas formas de agresión en el bienestar de los trabajadores. Además, estudia el efecto que tiene realizar actividades de recuperación que ayuden a las personas a desconectar de los problemas relacionados con el trabajo.

\section{Conclusiones}

El planteamiento metodológico de los estudios de diario está permitiendo nuevas perspectivas en el análisis del flujo con-

\section{Referencias}

Abad, F. J., Olea, J., Ponsoda, V. y García, C. (2011). Medición en ciencias sociales y de la salud. Madrid: Síntesis.

Almagor, M., y Ben-Porath,Y. S. (1989). The two-factor model of self-reported mood: A cross-cultural replication. Journal of Personality Assessment, 53(1), 1021.

Andersson, L. M., y Pearson, C. M. (1999). Tit for tat? The spiraling effect of incivility in the workplace. Academy of Management Review, 24(3), 452-471. Doi: 10.5465/AMR.1999.2202131

Balatsky, G., y Diener, E. (1993). Subjective well-being among Russian students. Social Indicators Research, 28(3), 225-243. Doi: 10.1007/BF01079019

Ben-Zur, H., y Yagil, D. (2005). The relationship between empowerment, aggressive behaviors of customers, coping, and burnout. European Journal of Work and Organizational Psychology, 14(1), 81-99. Doi: 10.1080/13594320444000281

Bryk, A. S., y Raudenbush, S. W. (1992). Hierarchical linear models: Application and data analysis methods. Newbury Park, CA: Sage.

Cortina, L. M., Kabat-Farr, D., Leskinen, E. A., Huerta, M., y Magley, V. J. (2013). Selective Incivility as Modern Discrimination in Organizations: Evidence and Impact. Journal of Management, 39(6), 1579-1605. Doi: $10.1177 / 0149206311418835$ tinuo de datos. Este diseño metodológico arroja un mayor control en los registros de medida y permite obtener evaluaciones en el contexto natural de trabajo, de suma importancia en el ámbito de la salud laboral. El estudio muestra el efecto interactivo de los estados emocionales y las situaciones organizacionales que experimenta un trabajador desde la mañana hasta la noche dentro de un mismo día, a lo largo de una semana de trabajo. De este modo, se observa cómo afectan las dinámicas de agresión laboral vividas durante el día de trabajo en la salud emocional de la persona, y en qué medida recuperarse de estas situaciones laborales agresivas durante el tiempo libre de la tarde, amortigua su efecto negativo y aumenta el estado de ánimo positivo de la noche. Específicamente, se observa que no todas las experiencias de recuperación son igualmente protectoras, por ejemplo, en este estudio las más significativas fueron la relajación, que tiene un efecto directo y de moderación en el afecto negativo de la noche; y la realización de actividades enriquecedoras, con un efecto directo sobre el afecto positivo. Además, la desconexión psicológica juega un papel importante como moderador del agotamiento emocional sobre el afecto positivo de la noche.

La investigación pone en evidencia la importancia que tiene identificar y erradicar estas situaciones tóxicas de trabajo para la salud emocional de la persona y para prevenir situaciones de discriminación laboral. Recientes investigaciones están poniendo de manifiesto cómo estas formas de agresión pueden actuar como un factor de discriminación laboral que desencadena una espiral de comportamientos incívicos en el trabajo con consecuencias negativas para los trabajadores y la organización (Cortina y cols., 2013). Por lo tanto, generar un cambio en las dinámicas laborales negativas es el primer paso para construir una organización saludable, así como potenciar las prácticas de autocuidado del trabajador.

Agradecimientos.- Proyecto Financiado por el Ministerio de Economía y Competitividad FEM2012-34692

Cortina, L. M., Magley, V. J., Williams, J. H., y Langhout, R. D. (2001). Incivility in the Workplace: Incidence and Impact. Journal of Occupational Health Psychology, 1, 64-80. Doi: 10.1037/1076-8998.6.1.64

Dimotakis, N., Scott, B. A., y Koopman, J. (2011). An experience sampling investigation of workplace interactions, affective states, and employee well-being. Journal of Organizational Behavior, 32(4), 572-588. Doi: 10.1002/job.722

Garrosa, E., Rainho, C., Moreno-Jimenez, B. y Monteiro, M. J. (2010). The relationship between job stressors, hardy personality, coping resources and burnout in a sample of nurses: a correlational study at two time points. International Journal of Nursing Studies, 47(2), 205-215. Doi: 10.1016/j.ijnurstu.2009.05.014

Grandey, A., Kern, J. H., y Frone, M. R. (2007). Verbal Abuse From Outsiders Versus Insiders: Comparing Frequency, Impact on Emotional Exhaustion, and the Role of Emotional Labor. Journal of Occupational Health Psychology, 12(1), 63-79. Doi: 10.1037/1076-8998.12.1.63

Grandey, A., Dickter, D., y Sin, H. P. (2004). The customer is not always right: Customer verbal aggression toward service employees. Journal of Organizational Behavior, 25(3), 397-418. Doi: 10.1002/job.252 
Hahn, V. C., Binnewies, C., Sonnentag, S., y Mojza, E. J. (2011). Learning How To Recover From Job Stress: Effects of a Recovery Training Program on Recovery, Recovery-Related Self-Efficacy, and Well-Being. Journal of Occupational Health Psychology, 16(2), 202-216. Doi: 10.1037/a0022169

Hershcovis, M. S., y Barling, J. (2010). Towards a multi-foci approach to workplace aggression: A meta-analytic review of outcomes from different perpetrators. Journal of Organizational Behavior, 31(1), 24-44. Doi: 10.1002/job.621

Hox, J. J., y Robers, J. K. (2011). Handbook of advanced multilevel analyses. New York, NY: Routledge.

Kabat-Farr, D., y Cortina, L. M. (2012). Selective incivility: Gender, race, and the discriminatory workplace. En S. Fox y T. R. Lituchy (Eds.), Gender and the dysfunctional workplace (pp. 120-134). Northampton, MA: Edward Elgar Publishing.

Lim, S., Cortina, L. M., y Magley, V. J. (2008). Personal and workgroup incivility: Impact on work and health outcomes. Journal of Applied Psychology, 93(1), 95107. Doi: 10.1037/0021-9010.93.1.95

Maslach, C., y Jackson, S. E. (1986). Maslach bumout inventory (2nd ed.). Palo Alto, CA: Consulting Psychologists Press.

Nägel, I. J., y Sonnentag, S. (2013). Exercise and sleep predict personal resources in employees' daily lives. Applied Psychology: Health And Well-Being, 5(3), 348368. Doi: 10.1111/aphw.12014

Ohly, S., Sonnentag, S., Niessen, C., y Zapf, D. (2010). Diary Studies in Organizational Research. An Introduction and Some Practical Recommendations. Journal of Personnel Psychology, 9(2), 79-93. Doi: 10.1027/1866-5888/a000009

Rashbash, J., Browne, W., Healy, M., Cameron, B., y Charlton, C. (2000). MLwiN (Version 1.10.006): Interactive software for multilevel analysis. Multilevel Models Project, Institute of Education. University of London: London.

Ryff, C. D., y Singer, B. H. (2008). Know thyself and become what you are: A eudaimonic approach to psychological well-being. Journal of Happiness Studies, 9(1), 13-39. Doi: 10.1007/s10902-006-9019-0

Sanz-Vergel, A. I., Demerouti, E., Moreno-Jiménez, B., y Mayo, M. (2010). Work-family balance and energy: A day-level study on recovery conditions. Journal of Vocational Behavior, 76(1), 118-130. Doi: 10.1016/j.jvb.2009.07.001

Sanz-Vergel, A. I., Sebastián, J., Rodríguez Muñoz, A., Garrosa Hernández, E., Moreno-Jiménez, B., y Sonnentag, S. (2010). Adaptación del "Cuestionario de Experiencias de Recuperación" a una muestra española. Psicothema, 22(4), 990-996.

Schat, A. C., y Kelloway, E. K. (2000). Effects of perceived control on the outcomes of workplace aggression and violence. Journal of Occupational Health Psychology, 5(3), 386-402. Doi: 10.1037/1076-8998.5.3.386
Sonnentag, S. (2011). Recovery From Fatigue: The Role of Psychological Detachment. En P. L. Ackerman (Ed.), Cognitive fatigue: Multidisciplinary perspectives on current research and future applications (pp. 253-272). Washington, DC, US: American Psychological Association.

Sonnentag, S., Kuttler, I., y Fritz, C. (2010). Job stressors, emotional exhaustion, and need for recovery: A multi-source study on the benefits of psychological detachment. Journal of Vocational Behavior, 76(3), 355-365. Doi: 10.1016/j.jvb.2009.06.005

Sonnentag, S., y Fritz, C. (2007). The recovery experience questionnaire: Development and validation of a measure for assessing recuperation and unwinding from work. Journal of Occupational Health Psychology, 12(3), 204-221. Doi: 10.1037/1076-8998.12.3.204

Spector, P. E., y Jex, S. M. (1998). Development of four self-report measures of job stressors and strain: Interpersonal conflict at work scale, organizational constraints scale, quantitative workload inventory, and physical symptoms inventory. Journal of Occupational Health Psychology, 3(4), 356-367. Doi: 10.1037/1076-8998.3.4.356

Thompson, E. R. (2007). Development of an internationally reliable shortform of the Positive and Negative Affect Schedule (PANAS). Journal of CrossCultural Psychology, 38(2), 227-242. Doi: 10.1177/0022022106297301

Watson, D., y Clark, L. A. (1984). Negative Affectivity: The disposition to experience aversive emotional states. Psychological Bulletin, 96(3), 465-490. Doi: 10.1037/0033-2909.96.3.465

Watson, D., Clark, L. A., y Tellegen, A. (1988). Development and validation of brief measures of positive and negative affect: The PANAS scales. Journal of Personality and Social Psychology, 54(6), 1063-1070. Doi: 10.1037/00223514.54.6.1063

Wharton, A. S. (1993). The Affective Consequences of Service Work: Managing Emotions on the Job. Work and Occupations, 20(2), 205-232. Doi: 10.1177/0730888493020002004

Xanthopoulou, D., Bakker, A. B., Demerouti, E., y Schaufeli, W. B. (2009). Work engagement and financial returns: A diary study on the role of job and personal resources. Journal of Occupational and Organizational Psychology, 82(1), 183 200. Doi: 10.1348/096317908X285633

(Articulo recibido: 25-10-2012; revisión recibida: 11-9-2013; aceptado: 20-1-2014) 